\title{
Análise das atividades biológicas dos venenos de Philodryas olfersii (Lichtenstein) e P. patagoniensis (Girard) (Serpentes, Colubridae)
}

\author{
Marisa M. T. da Rocha \& Maria de F. D. Furtado
}

Laboratório de Herpetologia, Instituto Butantan. Avenida Vital Brasil 1500, Butantan, 05503-900 São Paulo, São Paulo, Brasil.E-mail: bahrocha@uol.com.br; fatifurtado@butantan.gov.br

\begin{abstract}
Analysis of biological activities from Philodryas olfersii (Lichtenstein) and P. patagoniensis (Girard) venoms (Serpents, Colubridae). Philodryas olfersii (Lichtenstein, 1823) and P. patagoniesis (Girard, 1857) are species of colubrid snakes of the opisthoglyphous series restricted to South America. Several accidents caused by these snakes have been reported and they are mainly characterized by marked effects at the site of bite, such as pain, swelling and hemorrhage. Such accidents are similar to those caused by snakes of the genus Bothrops, and thereby patients bitten by Philodryas sp. are frequently treated with Bothrops antivenom. Since few studies have dealt with the characterization of these venoms, our aim was to study the venoms of $P$. olfersii and $P$. patagoniensis. They presented protein contents between 75 and $90 \%$. No defibrinogenating activity could be detected when tested in mice. Experimental envenomation induced by $P$ olfersii or $P$. patagoniensis evoked intense pain symptoms in mice, but the venom of $P$. patagoniensis was more active. Both venoms presented minimum edematogenic doses around $1 \mu \mathrm{g} /$ mouse, peaking at $30 \mathrm{~mm}$. The onset of hemorrhagic activity was rapid, and their minimum hemorrhagic doses were alike. The toxic activity of both venoms was similar, showing values around $60.0 \mu \mathrm{g} / \mathrm{mouse}$, which are comparable to those of Bothrops Wagler, 1824 venoms.
\end{abstract}

KEY WORDS. Local and systemic activities; toxics secretions; Xenodontinae.

RESUMO. Philodryas olfersii (Lichtenstein, 1823) e P. patagonienis (Girard, 1857) são serpentes colubrídeas da série opistóglifa, restritas à América do Sul. Vários acidentes ocasionados por estas serpentes têm sido relatados, caracterizando-se por ação local importante: dor, edema e hemorragia. É um acidente muito semelhante àquele causado por serpentes do gênero Bothrops Wagler, 1824 e muitas vezes os pacientes são tratados com soro antibotrópico. Poucos estudos tratam da caracterização destes venenos, assim tivemos como objetivo de trabalho o estudo dos venenos de $P$. olfersii e $P$. patagonienis. Os venenos apresentaram teor de proteínas entre 75 e $90 \%$. $A$ atividade desfibrinante não foi detectada quando testada em camundongos. $O$ quadro de dor causado pelo envenenamento experimental, em camundongos, mostrou que os venenos de $P$. olfersii e $P$. patagoniensis causaram intensa reatividade, sendo que o veneno de $P$. patagoniensis foi o mais ativo. Ambos os venenos apresentaram dose mínima edematogênica em torno de $1 \mu \mathrm{g}$ / camundongo com ação máxima em 30 minutos. A ação hemorrágica se instalou rapidamente, com doses mínimas semelhantes. As atividades tóxicas foram semelhantes, com valores em torno de 60,0 $\mu \mathrm{g} /$ camundongo, comparáveis aos venenos botrópicos.

PALAVRAS-CHAVE. Atividades locais e sistêmicas; secreções tóxicas; Xenodontinae.

Atualmente são descritas cerca de 2900 espécies de serpentes sendo que as da família Colubridae compreendem a mais de $65 \%$ de todas as serpentes existentes no mundo (Franco 2003).

A filogenia da família Colubridae proposta por KARDONG (2002) mostra uma relação próxima dos Xenodontinae com Elapidae, sendo este resultado corroborado por estudos de seqüência de DNA mitocondrial e RNA ribossomal (KNIGHT \& Mindell 1994, Lawson et al. 2005), dados de estrutura molecular de toxinas (Fry et al. 2003 a,b) e morfológicos (JACKSON 2003).
Cerca de 30 a $40 \%$ dos colubrídeos apresentam glândula de Duvernoy, homóloga às verdadeiras glândulas de veneno das serpentes proteróglifas e solenóglifas (Kochva 1963, GyGAX 1971, Ovadia 1984), as quais produzem secreções que consistem de enzimas, várias toxinas e outros compostos (MACKESSY 2002, Fry et al. 2003b).

Os acidentes ofídicos constituem um dos maiores problemas de saúde pública na América Latina (CAmpbell \& Lamar 1989, GutiérRez \& Lomonte 2003, WARrell 2004), e as serpentes peçonhentas são consideradas os principais agentes etiológicos 
destes. Contudo, levantamentos recentes têm demonstrado que cerca de 20 a $40 \%$ dos acidentes ofídicos no Brasil são causados por serpentes colubrídeas (Rosenfeld 1971, Silveira \& Nishioka 1992, Carvalho \& Nogueira 1998, Santos-Costa et al. 2001, SALOMÃo et al. 2003), sendo os principais gêneros Helicops Wagler, 1830, Oxyrhopus Wagler, 1830, Thamnodynastes Wagler, 1830 e Philodryas Wagler, 1830 (SANTOS-Costa et al. 2001, PUorTo \& FranCA 2003).

Embora a maioria dos registros não declare sérias conseqüências nos acidentes causados por serpentes opistóglifas, vários relatos ressaltam a importância de suas toxinas. Casos descrevendo a gravidade dos acidentes causados pelas xenodontíneas Philodryas olfersii (Lichtenstein, 1823) e Philodryas patagoniensis (Girard, 1857) em humanos têm sido descritos (Amaral 1921, Nickerson \& Henderson 1976, Silva \& Buononato 1983, Nishioka \& Sileira 1994, Araújo \& Santos 1997, Rocha et. al. 2003), assim como a ocorrência de óbito (SALOMÃo \& Di Bernardo 1995).

Os envenenamentos causados por espécies de Philodryas caracterizam-se por manifestações locais como dor, edema, eritema, equimose e linfodenopatia regional, com coagulação normal (Ribeiro et al. 1999).

Devido às semelhanças dos quadros fisiopatológicos, estes acidentes podem ser identificados como sendo envenenamentos botrópicos (Fan \& Cardoso 1995, França \& Málaque 2003) e muitas vezes soro antibotrópico tem sido administrado (Nishioka \& Silveira 1994, Ribeiro et al. 1994, 1999, Araújo \& SANTOS 1997).

Os venenos de $P$. olfersii e $P$. patagoniensis contém enzimas com diferentes atividades biológicas, entre elas, proteolítica, fibrin(ogen)olítica, hemorrágica e edematogênica, sendo desprovidos de enzimas do tipo-trombina, procoagulantes, fosfolipase $A_{2}$ e agregação plaquetária (Assakura et al. 1992, Prado-Franceschi et al. 1996, Acosta et al. 2003a, b, Реichoto et al. 2004, 2005).

AssaKURA et al. (1994) isolaram cinco diferentes proteinases fibrin(ogen)olíticas de veneno de P. olfersii, sendo elas as Pofibc ${ }_{1}$ $\mathrm{C}_{2}, \mathrm{C}_{3}$, H e S. Além disto, Prado-Franceschi et al. (1998) isolaram e caracterizaram uma fração miotóxica com peso molecular de 20 kDa, sem ação fosfolipásica do veneno desta espécie.

A despeito da grande diversidade de serpentes opistóglifas brasileiras é escasso o conhecimento das propriedades bioquímicas e farmacológicas de seus venenos, e considerando a importância dos acidentes ofídicos causados pelas serpentes do gênero Philodryas tivemos como objetivo de trabalho, estudar as ações biológicas dos venenos das espécies em questão. Philodryas olfersii com distribuição abrangendo os países da América Cisandina (Colômbia, Venezuela, Guiana, Brasil, Uruguai e norte da Argentina). No Brasil, esta espécie é encontrada em partes da região Nordeste e Centro-Oeste e em toda região Sudeste e Sul (Peter \& Orejas-Miranda 1970, Vanzolini 1986). Philodryas patagoniensis restrita a latitudes mais elevadas, ocorrem na Bolívia, Paraguai, Argentina e Uruguai e no Brasil nas regiões Nordeste, Centro-Oeste e em toda região Sudeste e Sul (Peter \& OrejasMiranda 1970).

\section{MATERIAL E MÉTODOS}

\section{Venenos}

Serpentes adultas da subespécie Philodryas olfersii olfersii (Fig. 1) e da espécie Philodryas patagoniensis (Fig. 2), medindo de 300 a $1200 \mathrm{~mm}$, foram mantidas em cativeiro, nos Biotérios de manutenção do Laboratório de Herpetologia do Instituto Butantan, em gaiolas individuais, recebendo água à vontade e alimentação constituída de camundongos, a cada 30 dias. Três semanas após a alimentação, as serpentes foram inoculadas via intraperitoneal (i.p.) com solução de pilocarpina diluída em solução salina $0,85 \%$, na concentração de $10 \mathrm{mg} / \mathrm{kg}$ (Rosenberg et al. 1985) e o veneno extraído por meio da introdução de suas presas em micropipetas de $100 \mu \mathrm{l}$ (FerLan et al. 1983). As amostras foram liofilizadas e armazenadas a $-20^{\circ} \mathrm{C}$ até o momento do uso.

\section{Animais}

Camundongos Swiss, machos, pesando de 18 a $22 \mathrm{~g}$ e ratos Wistar, machos pesando de 230 a 250 g provenientes do Biotério Central do Instituto Butantan foram utilizados. Todos os ensaios foram realizados segundo as normas da Comissão de Ética de Utilização de Animais Experimentais do Instituto Butantan e sob as condições estabelecidas no processo de número 009/2000.

\section{Determinação do teor de Proteínas}

A dosagem de proteínas foi determinada através do teor de proteínas totais segundo o método de Lowry et al. (1951), modificado por MARKwell et al. (1978). Albumina bovina foi utilizada como padrão. A leitura das D.O. $(660 \mathrm{~nm})$ foi realizada em espectrofotômetro Micronal-Modelo B383 utilizando-se como "branco" a amostra a qual o veneno não foi adicionado. A quantidade de proteínas foi expressa em microgramas de proteína por miligrama de veneno. Foram realizados testes em triplicata.

\section{Atividade desfibrinante}

Para a atividade desfibrinante dos venenos foi utilizado o método de GeNé et al. (1989), onde grupos de 04 camundongos albinos, não isogênicos, pesando de 18 a 22 g, foram injetados pela via intravenosa (i.v.), na cauda, com $100 \mu \mathrm{l}$ das soluções de venenos, diluídos em salina $0,85 \%$ estéril, nas concentrações de 10 e $20 \mu \mathrm{g}$. Após uma hora, os animais foram sangrados via plexo orbital, o material incubado a $37^{\circ} \mathrm{C}$ e observado o tempo de coagulação do sangue.

\section{Atividade Nociceptiva}

Para a determinação da atividade nociceptiva (dor) dos venenos de $P$. olfersii e $P$. patagoniensis camundongos $(\mathrm{n}=10)$ foram injetados na região intraplantar (s.c) da pata posterior direita com 1,0 $\mu \mathrm{g} / 50 \mu \mathrm{l}$ de solução salina estéril; como controle animais foram injetados somente com salina $0,85 \%$ estéril. Os animais foram colocados individualmente sob funis de vidro, localizados sobre uma superfície refletora para facilitar a observação. Em seguida, foi medida a reatividade dos animais, considerada como o tempo gasto, em segundos, em que os animais, 

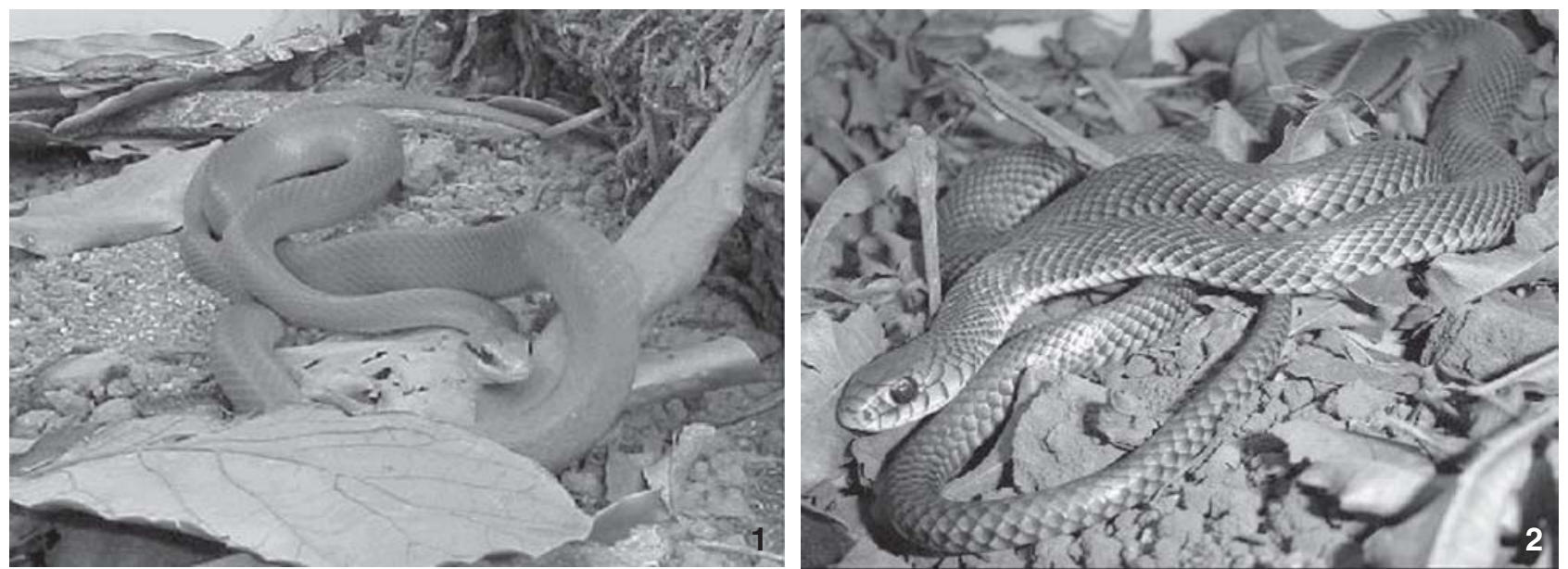

Figuras 1-2. (1) Philodryas olfersii exemplar adulto; (2) P. patagoniensis exemplar adulto. Fotos de Silvia R. Travaglia-Cardoso.

lambiam ou mordiam a pata injetada, durante 30 minutos de avaliação experimental (HunsKAAR et al. 1985). Os resultados foram expressos como médias dos tempos de reatividade dos animais \pm erro padrão, em dois diferentes experimentos.

\section{Atividade edematogênica}

Para a determinação da atividade edematogênica dos venenos foi utilizado o método de Yamakawa et al. (1976), com modificações. Inicialmente foi estabelecida a cinética da atividade edematogênica, onde grupos de 06 camundongos albinos, não isogênicos, pesando de 18 a 22 g, foram injetados, s.c., no coxim da pata direita (experimental), com $50 \mu \mathrm{l}$ da solução de veneno diluído em salina estéril $0,85 \%$ e a pata contralateral (controle) injetada somente com salina estéril. As espessuras dos coxins foram medidas com espessímetro (Mituttoyo - precisão 0,01 mm) nos tempos de 15 minutos, 30 minutos, 1, 2, 3, 6 e 24 horas. O edema foi expresso pela diferença entre os aumentos de espessura nos coxins das patas experimental e controle, dividida pelos valores das espessuras das patas controle, multiplicado por 100. Para a determinação da Dose Mínima Edematogênica (DME), que é definida como a menor quantidade de veneno necessária para induzir 30\% de aumento máximo da espessura da pata "experimental", os animais foram tratados como descrito acima se utilizando soluções dos venenos em concentrações variadas e medindo-se as espessuras dos coxins 30 minutos após a inoculação (tempo determinado pela cinética de ação).

\section{Atividade hemorrágica}

$\mathrm{Na}$ atividade hemorrágica utilizou-se o método descrito por Kondo et al. (1960), modificado por THEAKSTON \& REID (1983). Para a determinação do tempo de ação (cinética) dos venenos quanto ao desenvolvimento da atividade hemorrágica, alíquotas de $0,1 \mathrm{ml}$ de uma solução de $20 \mu \mathrm{g}$ dos venenos de $P$. olfersii e $P$. patagoniensis foram injetadas, i.d., na pele previamente depilada do dorso de ratos machos adultos, pesando de 230 a 250 g, sob anestesia leve de $\mathrm{CO}_{2}$. Após os tempos de 30 minutos, 1, 2, 3, 6 e 16 horas, os animais foram sacrificados, e a pele do dorso removida para a determinação da área de lesão hemorrágica, através do cálculo dos diâmetros médios para cada tempo.

Para a determinação da Dose Mínima hemorrágica (DMH), que é definida como a menor quantidade de veneno em microgramas que, quando injetada na pele do dorso de ratos, produz uma lesão hemorrágica de $10 \mathrm{~mm}$ de diâmetro, grupos de seis ratos foram tratados como descrito acima, com soluções dos venenos de $P$. olfersii e $P$. patagoniensis nas concentrações de 15 a $25 \mu \mathrm{g}$ e de 15 a $31 \mu \mathrm{g}$ sendo os animais sacrificados 2 e 4 horas, respectivamente (tempo determinado pela cinética de ação).

\section{Atividade Miotóxica}

Para a avaliação da atividade miotóxica foi utilizado o método de quantificação dos níveis séricos da enzima creatinoquinase (CK), que é liberada após lesão muscular, conforme descrito por GuTiérRez et al. (1980). Grupos de cinco camundongos foram injetados com $50 \mu$ de soluções dos venenos, na concentração de $30 \mu \mathrm{g}$, pela via intramuscular (i.m.), no músculo gastrocnêmio, e os animais controle foram inoculados somente com salina $0,85 \%$ estéril. Os animais foram sangrados via plexo ocular nos tempos de 1, 3, 6, 12 e $24 \mathrm{~h}$. O soro, obtido por centrifugação, foi testado utilizando-se o "kit da Sigma Diagnostics (USA) - Creatine Phosfokinase (CK) - Quantitative Colorimetric Detremination in Serum or Plasma.

\section{Atividade Necrosante}

Foi utilizado o método descrito por Kondo et al. (1960), onde estipula-se a Dose Mínima Necrosante, que é definida como a menor quantidade de veneno, em microgramas que, quando injetada i.d. na pele do dorso de ratos, produz, em 24 h, uma lesão necrótica de $5 \mathrm{~mm}$ de diâmetro. Grupos de seis ratos foram injetados (conforme descrito na atividade hemorrágica), com soluções dos venenos de $P$. olfersii e $P$. 
patagoniensis, nas concentrações de 40, 50, 60, 70, 80 e $90 \mu \mathrm{g}$ e avaliadas as áreas de lesões.

\section{Dose Letal (DL50)}

Grupos de seis camundongos foram injetados com diferentes concentrações dos venenos de cada uma das espécies de serpentes. O número de animais mortos para cada dose foi observado em 48 horas. O cálculo foi baseado no número total de camundongos mortos por dose de veneno durante os experimentos (Villarroel et al. 1978) e analisados através do método "Full Probit Analysis" (Finney 1971).

\section{Análises estatísticas}

Os resultados foram expressos como média \pm erro padrão da média (E.P.M.) das amostras analisadas. As comparações entre os grupos amostrais foram realizadas através da análise de variância (ANOVA) e o teste $t$ de Student para a comparação entre as espécies.

Para a análise estatística das DL50\% foi utilizada a metodologia descrita por Litchireld \& WiLcoxon (1949), utilizando-se o programa estatístico STATA ${ }^{\mathrm{TM}}$, versão 8.0.

\section{RESULTADOS}

A análise do teor de proteínas dos venenos de P. olfersii e P. patagoniensis demonstrou uma variação entre 75 e $90 \%$, respectivamente.

A atividade desfibrinante, testada nas doses de 10 e 20 $\mu \mathrm{g} /$ camundongo, para os venenos de $P$. olfersii e $P$. patagoniensis, injetados via intravenosa, não causou alteração do tempo de coagulação do sangue dos animais $(n=4)$.

$\mathrm{Na}$ avaliação do efeito edematogênico foi observado que os venenos têm ação muito rápida, entre cinco e dez minutos após a injeção o edema evolui, sendo que sua ação máxima ocorre em 30 minutos, mantendo-se estável até a quarta hora (Fig. 3). As Doses Mínimas Edematogênicas foram semelhantes com valores de 1,1 $\pm 0,07 \mu \mathrm{g}$ para o veneno de $P$. olfersii e $0,9 \pm 0,08 \mu \mathrm{g}$ para o veneno de $P$. patagoniensis (médias \pm E.P.M.; $\mathrm{n}=6$; $\mathrm{p}>$ 0,05; F: 1.306) (Fig. 4).

A inoculação intradérmica em dorso de ratos, com os venenos $P$. olfersii e $P$. patagoniensis produziu hemorragia, com pico de ação em quatro e duas horas (Fig. 5), com Doses Mínimas Hemorrágicas iguais e valores de $24,1 \pm 2,8 \mu \mathrm{g}$ /rato e 26,9 $\pm 2,5 \mu \mathrm{g} /$ rato respectivamente (média \pm E.P.M.; $\mathrm{n}=6$; $\mathrm{p}>0,05$; F: 1.254) (Fig. 6).

Para a avaliação da atividade nociceptiva, camundongos $(\mathrm{n}=10)$ foram injetados na região intraplantar com $1 \mu \mathrm{g}$ dos venenos, com os animais observados por um período de $30 \mathrm{mi}-$ nutos. Os resultados mostraram que os venenos de $P$. olfersii e $P$. patagoniensis apresentaram valores quanto à nocicepção, de 53 , $9 \pm 10,4$ seg. e 99,7 $\pm 13,5$ seg. (média \pm E.P.M.; $n=10 ; p<0,05$; F: 1.735), sendo que o veneno de $P$. patagoniensis foi o mais ativo (Fig. 7).

Os venenos de $P$. olfersii e $P$. patagoniensis nas doses de $50,60,70,80$ e $90 \mu \mathrm{g}$, quando injetados i.d. em dorso de ratos, provocaram lesão necrótica em 24 horas. As Doses Mínimas Necrosantes (DMN) foram significativamente diferentes, sendo que o veneno de $P$. olfersii apresentou DMN de 79,1 $\pm 3,9$ $\mu \mathrm{g} / \mathrm{rato}$ e $P$. patagoniensis $=63,5 \pm 4,6 \mu \mathrm{g} / \mathrm{rato}$ (média \pm E.P.M.; $\mathrm{n}=6 ; \mathrm{p}<0,05 ; \mathrm{F}: 1.391$ ) (Fig. 8).

A atividade miotóxica dos venenos foi avaliada através da injeção intramuscular (i.m.) (30 $\mathrm{\mu g} /$ camundongo) e a determinação da liberação de Cretino-quinase (CK) plasmática, enzima liberada após lesão muscular. Como apresentado na figura 9, os animais injetados com os venenos não mostraram alterações significativas dos níveis de CK plasmática quando comparados com o grupo controle (injetados com salina). O pico de ação ocorreu três horas após a injeção e retornando aos níveis basais a partir da $12^{\mathrm{a}}$ hora, e os valores para a liberação de CK foram de 25,8 $\pm 5,1 \mathrm{U} / \mathrm{ml}$ (P. olfersii), 22,4 $\pm 3,2 \mathrm{U} / \mathrm{ml}(P$. patagoniensis) e $17,9 \pm 2,5$ (grupo controle) em 180 minutos (média \pm E.P.M.; $\mathrm{n}=5 ; \mathrm{p}>0,05 ; 2.540$ ).

A atividade letal foi semelhante nos venenos de ambas as espécies. P. olfersii apresentou a Dose Letal 50\% de 62,43 $\mu \mathrm{g} /$ camundongo e $P$. patagoniensis de 58,85 $\mu \mathrm{g} /$ camundongo, com limite de confiança 95\% (Fig. 10).

\section{DISCUSSÃO}

Diferentes métodos para obtenção dos venenos de colubrídeos têm sido utilizados (WeINSTEIN \& KARDONG 1994, MACKESSY 2002), após cerca de 1000 extrações, concluímos que a técnica comum de extração, como realizada em serpentes proteróglifas e solenóglifas, com a simples compressão da glândula de veneno, não é eficiente devido à natureza do aparato inoculador das serpentes opistóglifas. Nas glândulas Duvernoy os grânulos de secreção estão estocados na região interna das células, sendo liberados somente no momento do estímulo (Kochva 1987), desta forma a extração direta com capilares introduzidos nas presas (Ferlan et al. 1983) é o método mais eficaz, uma vez que a secreção estará livre de agentes contaminantes (MACKEssy 2002).

Os venenos de serpentes são misturas contendo múltiplos componentes, sendo que $90 \%$ são proteínas. Segundo Weinstern \& Kardong (1994), Hill \& Mackessy (2000) e Mackessy (2002), o conteúdo proteico dos venenos de colubrídeos é bastante variável podendo apresentar baixos níveis de proteínas e o material liofilizado contendo altas concentrações de componentes não proteicos (Vest et al. 1991, WeInstein et al. 1991). De modo geral existe uma variação entre 15 e 100\% dentre os venenos já estudados (Mackessy 2002). Os venenos de P. olfersii e P. patagoniensis apresentaram conteúdo de proteínas totais entre 75 e $90 \%$ respectivamente.

As proeminentes atividades edematogênica e hiperalgésica, características nestes acidentes, e seus rápidos desenvolvimentos são típicos sinais do processo inflamatório agudo, decorrente da ação de substâncias endógenas que são liberadas após o estímulo lesivo (Ohsaka 1979, Chacur et al. 2001, PradoFranceschi \& Hysloo 2002). Os venenos de P. olfersii e $P$. patagoniensis apresentaram, em camundongos, respostas hiper-

Revista Brasileira de Zoologia 24 (2): 410-418, junho 2007 

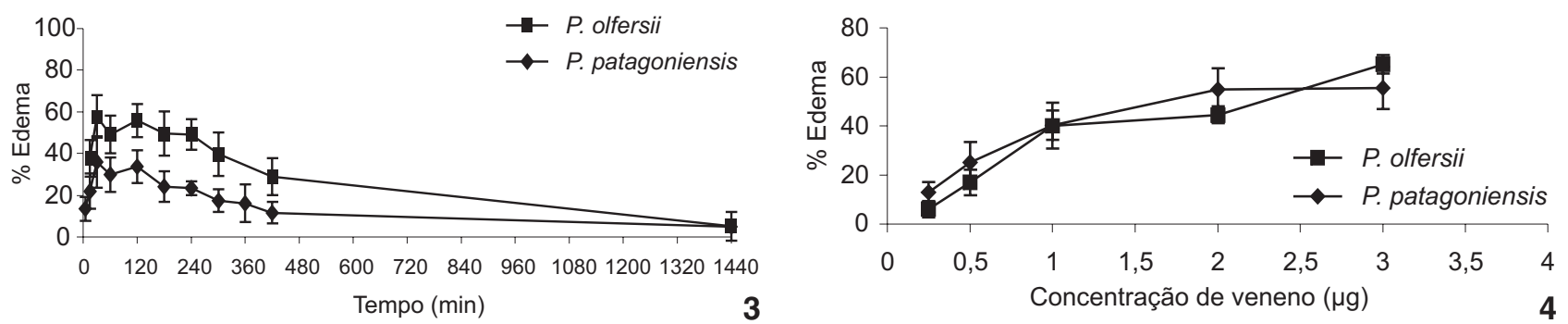

Figuras 3-4. (3) Cinética do edema após a injeção intraplantar de $3 \mu \mathrm{g}$ dos venenos de $P$. olfersii e $P$. patagoniensis; (4) Curva doseresposta da ação edematogênica dos venenos de P. olfersii e P. patagoniensis. Os dados representam a média \pm E.P.M. $(n=6)$.
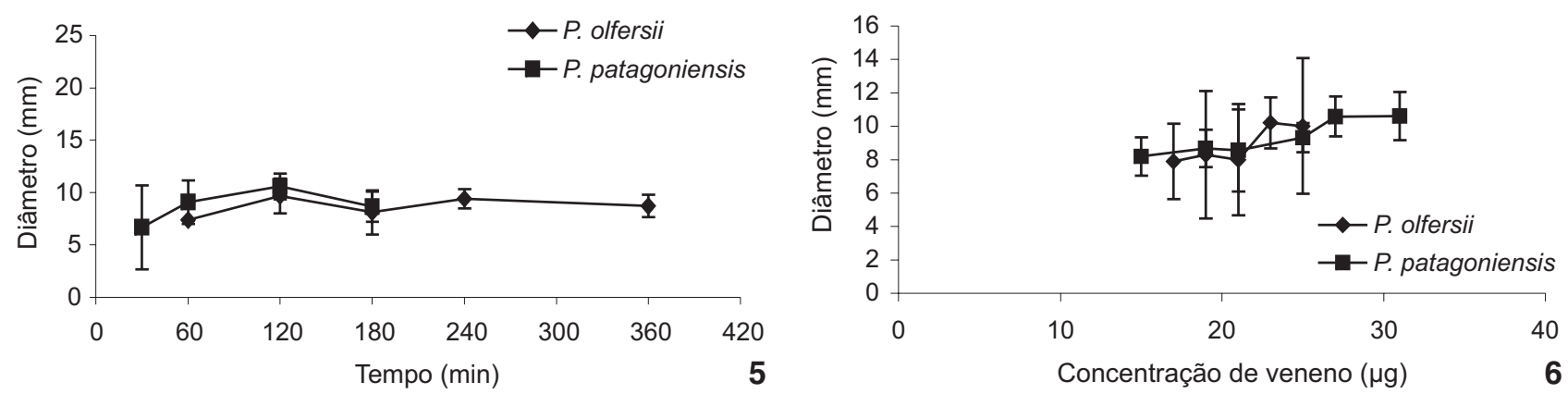

Figuras 5-6. (5) A cinética da atividade hemorrágica dos venenos de $P$. olfersii e $P$. patagoniensis foi avaliada após a injeção intradérmica de $20 \mu \mathrm{g}$ dos venenos. Os dados representam a média \pm E.P.M. ( $\mathrm{n}=6$ por tempo); (6) determinação da Dose Mínima Hemorrágica dos venenos de P. olfersii e P. patagoniensis.
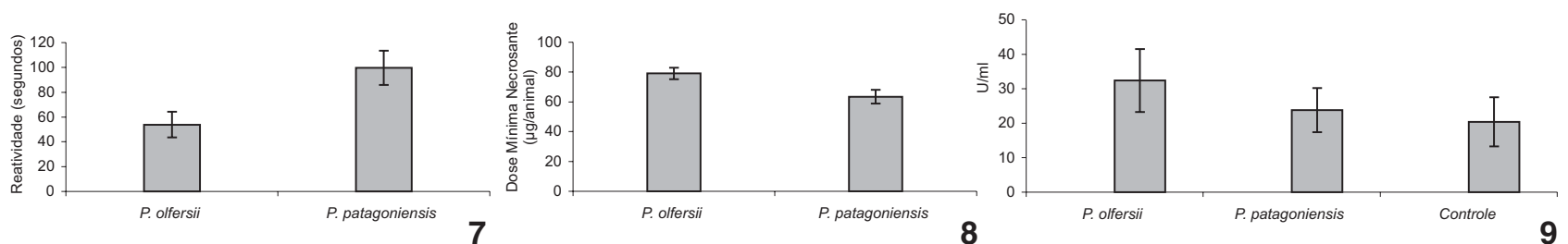

Figuras 7-9. (7) Atividade nociceptiva dos venenos de P. olfersii e P. patagoniensis na dose de $1 \mu \mathrm{g}$; (8) comparação das Doses Mínimas Necrosantes dos venenos de $P$. olfersii e P. patagoniensis; (9) comparação dos níveis plasmáticos de Cretino-quinase (CK) após a inoculação i.m. $(30 \mu \mathrm{g} / 50 \mu \mathrm{l})$ dos venenos de $P$. olfersii e P. patagoniensis. Os dados representam a média \pm E.P.M. de: (7) dez animais, (8) quatro animais; cinco animais.

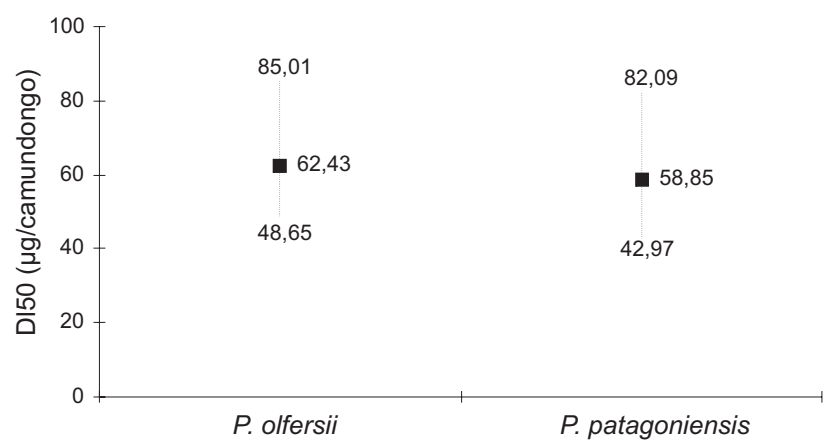

Figura 10. Representação gráfica das Doses Letais $50 \%$ dos venenos de $P$. olfersii e $P$. patagoniensis. As $\mathrm{DL}_{50} P$. olfersii e $P$. patagoniensis foram estatisticamente iguais (limite de confiança $95 \%$ e $p>0,05$ ). algésicas importantes, sendo que o veneno de $P$. patagoniensis foi mais ativo que o veneno de $P$. olfersii.

Assakura et al. (1992), Acosta et al. (2003 a, b) е Реiсното et al. (2004) determinaram Doses Mínimas Edematogênicas para os venenos de $P$. olfersii $(0,25$ e $0,31 \mu \mathrm{g})$ e $P$. patagoniensis $(0,26 \mu \mathrm{g})$, sendo mais edematogênico que alguns venenos botrópicos. Os resultados aqui apresentados mostraram que ambos os venenos apresentaram ação máxima em 30 minutos após a inoculação e as Doses mínimas foram mais elevadas, com valores de 1,1 $\mu \mathrm{g} /$ camundongo para o veneno de $P$. olfersii e $0,86 \mu \mathrm{g} / \mathrm{camundongo}$ para o veneno de $P$. patagoniensis, sendo que estas diferenças poderiam estar ligadas á variação geográfica (CHIPpAux et al. 1991).

A atividade hemorrágica dos venenos de viperídeos (SANChez et al. 1992, BJARnASON \& Fox 1994, GutiérReZ \& Rucavado

Revista Brasileira de Zoologia 24 (2): 410-418, junho 2007 
2000) e colubrídeos (Assakura et al. 1992, Mandelbaum et al. 1998, Acosta et al. 2003b) é atribuída, principalmente à atuação de metaloproteínases, enzimas proteolíticas com atividade dependente do íon zinco.

Assim como nos viperídeos, as hemorraginas presentes nos venenos de colubrídeos podem ter um papel semelhante com relação aos processos digestivos e a presença de atividade hemorrágica tem sido largamente indicada em várias espécies de colubrídeos (KornaliK et al. 1978, Vest 1988, NavarRete et al. 1999, Lemoine \& Rodrigues-Acosta 2003, Lemoine et al. 2004a, b, Rocha et al. 2006).

TANJONI et al. (2003) utilizando um anticorpo monoclonal anti-jararagina que reconhece um epítopo presente na região Cterminal do domínio desintegrina-like, uma metaloproteinase do veneno de Bothrops jararaca (Wied, 1824), demonstraram pela técnica de Dot Blot que estes anticorpos reagiram com os venenos de $P$. olfersii e $P$. patagoniensis, sugerindo que as metaloproteinases destes venenos apresentam epítopos comuns aos de B. jararaca. Corroborando tais achados, Rocha et al. (2006) demonstraram que o soro antibotrópico comercial apresentou reatividade cruzada sendo capaz de neutralizar a ação hemorrágica, e eficientemente a atividade tóxica dos venenos de $P$. olfersii e $P$. patagoniensis, com títulos de potência similares aos obtidos com o veneno de $B$. jararaca.

Os venenos de $P$. olfersii e $P$. patagoniensis apresentaram Doses Mínimas Hemorrágicas de 24,1 e 26,9 ㄱg/rato, respectivamente. Observa-se ainda que, estes são menos hemorrágicos do que aqueles de serpentes do gênero Bothrops Wagler, 1824 tais como: B. jararaca $(15,6 \mu \mathrm{g} / \mathrm{rato}), B$. cotiara (Gomes, 1913) (20,2 $\mu \mathrm{g} / \mathrm{rato})$ e B. alternatus Duméril, Bibron \& Duméril, 1854 $(14,4 \mu \mathrm{g} / \mathrm{rato})$ (FurTADo et al. 1991), analisados em 24 horas, sendo os de Philodryas avaliados nos tempos de 2 e 4 horas, com ação mais rápida.

As miotoxinas são toxinas responsáveis pelo dano muscular, podendo estar ligadas a uma ação fosfolipásica. Pouco se conhece sobre as miotoxinas dos venenos de colubrídeos e na maioria dos estudos estas toxinas têm sido parcialmente purificadas (Jansen 1987, Prado-Franceschi et al. 1996, 1998). Segundo Assakura et al. $(1992,1994)$ o veneno de P. olfersii apresenta baixa atividade fosfolipásica. Os resultados mostraram baixos níveis de liberação de creatino-quinase nos vários tempos testados, com pico de ação três horas após a inoculação e retornando aos níveis basais a partir da $12^{\mathrm{a}}$ hora. Entretanto, Acosta et al. (2003a, b) е Регсното et al. (2004) utilizando serpentes deste mesmo gênero do nordeste da Argentina, determinaram altos níveis desta enzima tardiamente entre 10 e 16 horas.

A atividade necrosante decorrente dos envenenamentos de serpentes viperídeas originam-se da ação de enzimas proteolíticas causando a destruição dos tecidos moles próximos ao local da picada (Rosenfeld 1971). Entre os envenenamentos causados por colubrídeos não tem sido descrita a atividade necrosante. Entretanto, Регсното et al. (2004) determinaram esta atividade em veneno de $P$. patagoniensis o qual apre- sentou DMN igual a $180,5 \mathrm{mg} /$ rato. Os resultados aqui apresentados mostraram que os venenos de P. olfersii (DMN $=79,1 \mu \mathrm{g} /$ rato $\pm 3,9)$ e $P$. patagoniensis $(\mathrm{DMN}=63,5 \mu \mathrm{g} / \mathrm{rato} \pm 4,6)$ são capazes de causar pronunciada dermonecrose, sendo o veneno de $P$. patagoniensis o mais ativo.

As doses letais das espécies $P$. olfersii e $P$. patagoniensis foram determinadas em $62,4 \mu \mathrm{g} /$ camundongo (Limites de confiança $95 \%=85,01-48,65$ ) e $58,8 \mu \mathrm{g} /$ camundongo (Limites de confiança $95 \%=82,09-42,97)$, respectivamente e, consideradas estatisticamente semelhantes, com nível de significância de $95 \%$. Apesar de menos tóxicos do que o veneno de $B$. jararaca $(24,7 \mu \mathrm{g} /$ camundongo e Limites de confiança 95\% $=23-26 \mu \mathrm{g} /$ camundongo) seus valores podem ser equiparados aos venenos de $B$. jararacussu Lacerda, 1884 (58,8 $\mu \mathrm{g} /$ camundongo e Limites de confiança $=52-67 \mu \mathrm{g} /$ camundongo) e $B$. alternatus $(67,5 \mu \mathrm{g} / \mathrm{camundongo}$ e Limites de confiança $=59-76 \mu \mathrm{g} / \mathrm{ca}-$ mundongo) (FurTADO et al. 1991).

Os venenos de $P$. olfersii e $P$. patagoniensis apresentam atividades biológicas semelhantes aos venenos botrópicos, sendo as ações locais menos intensas embora os tempos de desenvolvimento dos efeitos mais rápidos.

\section{AGRADECIMENTOS}

A Silvia R. Travaglia-Cardoso e Valdir Germano e aos biólogos Henrique Braz, Laura Narvaes, André Zelanis e Mônica F. Lopes.

\section{REFERÊNCIAS BIBLIOGRÁFICAS}

Acosta, O.; L.C. Leiva; M.E. Peichoto; S. Makuñak; P.T. Rúz; C. Gay \& L. Rey. 2003a. Edematogenic and myotoxic activities of the Duvernoy's gland secretion of Philodryas olfersii from the north-est region of Argentina. BIOCEL 27 (3): 363-370.

Acosta, O.; L.C. Leiva; M.E. Peichoto; S. Maruñak; P. Tebler \& L. Rey. 2003b. Hemorrhagic activity of the Duvernoy's gland secretion of the xenodontine colubrid Philodryas patagoniensis from the north-east region of Argentina. Toxicon 41: 10071012.

Amaral, A. 1921. Últimos trabalhos inéditos de J. Florencio Gomes: Duas novas espécies de Colubrídeos opisthoglyphos brasileiros (Philodryas oligolepis e Apostolepis longicaudata). Annais Paulista de Medicina e Cirurgia 9 (7/8): 157-221.

Araújo, M.E. \& A.C.M.C.A. Santos. 1997. Cases of human envenoming caused by Philodryas olfersii and Philodryas patagoniensis (Serpentes: Colubridae). Revista da Sociedade Brasileira de Medicina Tropical 30 (6): 517-519.

Assakura, M.T.; A.P.N. Reichl \& F.R. Mandelbaum. 1994. Isolation and characterization of five fibrin(ogen)olytic enzymes from the venom of Philodryas olfersii (green snake). Toxicon 32 (7): 819-831.

Assakura, M.T.; M.G. Salomão; G. Puorto\& F.R. Mandelbaum. 1992. Hemorrhagic, fibrinogenolytic and edema-forming activities of the venom of the colubrid snake Philodryas olfersii (green 
snake). Toxicon 30 (4): 427-438.

BJARNASON, J. \& J.W. Fox. 1994. Hemorrhagic metalloproteinases from snake venoms. Pharmacology \& Therapeutics 62: 325-372.

Campbell, J.A. \& W.W. Lamar. 1989. The Venomous Reptiles of Latin America. London, Comstok, $6^{\text {th }}$ ed., 425p.

Carvalho, M.A \& F. Nogueira. 1998. Serpentes da área urbana de Cuiabá, Mato Grosso: aspectos ecológicos e acidentes ofídicos associados. Cadernos de Saúde Pública 14: 753763.

Chacur, M.; G. Picolo; J.M. Gutiérrez; C.F.P. Teixeira \& Y. Cury. 2001. Pharmacological modulation of hyperalgesia induced by Bothrops asper (terciopelo) snake venom. Toxicon 39: 1173-1181.

Chippaux, J. P.; V. Williams and J. White. 1991. Snake venom variability: Methods of study, results and interpretation. Toxicon 29: 1279-1303.

Fan, H.W. \& J.L. Cardoso. 1995. Clinical toxicology of snake bites in South America, p. 667-688. In: J. MeIER \& J. White (Eds). Handbook of clinical toxicology of animal venoms and poisons. Boca Raton, CRC Press, 752p.

Ferlan, I.; A. Ferlan; T. King \& F.E. Russell. 1983. Preliminary studies on the venom of the colubrid snake Rhabdophis subminiatus (red-necked keelback). Toxicon 21 (4): 570-574.

Finney, P.J. 1971. Probit Analysis. Cambridge, Cambridge University Press, $3^{\text {rd }}$ ed., 333p.

França, F.O.S. \& C.M.S. Málaque. 2003. Acidente botrópico p. 72-86. In: J.L.C. Cardooso; F.O.S. França; F.H. Wen; C.M.S Málaque \& V. Haddad Jr (Eds). Animais peçonhentos no Brasil: biologia, clínica e terapêutica dos acidentes. São Paulo, Sarvier, FAPESP, 468p.

FRANCO, F.L. 2003. Origem e diversidade das serpentes, p. 13-32. In: J.L.C. Cardoso; F.O.S.França; F.H. Wen; C.M.S Málaque; V. HADDAD JR (Eds). Animais peçonhentos no Brasil: biologia, clínica e terapêutica dos acidentes. São Paulo, Sarvier, FAPESP, 468p.

Fry, B.G.; N.G. Lumsden; W. Wuster; J.C. Wickramaratna; W.C. Hodgson \& R.M. KINI. 2003a. Isolation of neurotoxin $(\alpha-$ colubritoxin) from a nonvenomous Colubrid: evidence for early origin of venom in snakes. Journal of Molecular Evolution 57: 446-452.

Fry, B.G.; W. Wüster; S.F.R. Ramjan; T. Jackson; P. Martelli \& M. KINI. 2003b. Analysis of Colubroidea snake venoms by liquid chromatography with mass spectrometry: evolutionary and toxicological implications. Rapid Communications in Mass Spectrometry 17: 2047-2062.

Furtado, M.F.D.; W. Dias da Silva \& G.M.D.D. Colleto. 1991. Controle de Qualidade dos Venenos Animais e dos Correspondentes Antivenenos. I Padronização dos métodos de ensaio das atividades bioquímicas e farmacológicas dos venenos de algumas espécies do gênero Bothrops e Crotalus usando amostras secas a temperatura ambiente ou liofilizadas. Memórias do Instituto Butantan 53 (2): 149-159.
Gené, J.A.; A. Roy; G. Rojas; J.M. GutiérRez \& L. Cerdas. 1989. Comparative study on coagulant, defibrinanting, fibrinolytic and fibrinogenolytic activities of Costa Rican crotaline snake venoms and their neutralization by a polyvalent antivenom. Toxicon 27 (8): 841-848.

GutiéRRez, J.M. \& A. RuCAVADo. 2000. Snake venom metalloproteinases: their role in the pathogenesis of local tissue damage. Biochimie 82: 841-850.

Gutiérrez, J.M. \& B. Lomonte. 2003. Efectos Locales en el Envenenamiento Ofídico en América Latina, p. 310-323. In: J.L.C. Cardooso; F.O.S.França; F.H. Wen; C.M.S Málaque; V. HADDAD JR (Eds). Animais peçonhentos no Brasil: biologia, clínica e terapêutica dos acidentes. São Paulo, Sarvier, FAPESP, 468p.

Gutiérrez, J.M.; O. Arroyo \& R. Bolaños. 1980. Mionecrosis, hemorragia y edema inducidos por el veneno de Bothrops asper en raton blanco. Toxicon 18: 603-610.

GygaX, P. 1971. Development, morphology and function of the venom gland (Duvernoy's gland) of Natrix tessellata. Acta Tropopica 28 (3): 226-274.

Hill, R.E. \& S.P. Mackessy. 2000. Characterization of venom (Duvernoy's secretion) from twelve species of colubrid snakes and partial sequence of four venom proteins. Toxicon 38: 1663-1687.

Hunskaar, S.; O.B. Fasmer \& K. Hole. 1985. Formain test in mice, a useful technic for evaluation mild analgesics. Journal of Neuroscience Methods 14: 769-785.

JACKSON, K. 2003. The evolution of venom-delivery systems in snakes. Zoological Journal of the Linnean Society 137: 337-354.

JANSEN, D.W. 1987. The myonecrotic effect of Duvernoy's gland secretion of the Thamnophis elegans vagrans. Journal of Herpetology 21: 81-83.

KARDONG, K.V. 2002. Colubrid snakes and Duvernoy's "venom" glands. Journal of Toxicology-Toxin Reviews 21: 1-19.

KNIGHT, A. \& D. Mindell. 1994. On the phylogenetic relationship of Colubrinae, Elapidae, and Viperidae and the evolution of front-fanged venom systems in snakes. Copeia 1994 (1): $1-9$.

Kochva, E. 1963. The development of the venom gland in the opisthoglyph snake Telescopus fallax with remarks on Thamnophis sirtalis (Colubridae, Reptilia). Copeia 1963 (2): 147-154

Kochva, E. 1987. The origin of snake and evolution of the venom apparatus. Toxicon 25 (1): 65-106.

Kondo, H.; S. Kondo; I. Ikesawa; R. Murata; A. Ohsaka. 1960. Studies of the quantitative method for determination of hemorrhagic activity of Habu snake venom. Japanese Journal of Medical Science and Biology 13: 43-51.

KornaliK, F.; E. TÁborKÁ \& D. Mebs. 1978. Pharmacological and biological properties of a venom gland extract from the snake Thelotornis kirtlandi. Toxicon 16: 535-542.

Lawson, R.; J.B. Slowinski; B.I. Crother \& F.T. Burbrink. 2005. 
Phylogeny of Colubroidea (Serpentes): New evidence from mitochondrial and nuclear genes. Molecular Phylogenetics and Evolution 37: 581-601.

Lemoine, K. \& A. Rodríguez-Acosta. 2003. Haemorrhagic, proteolytic and neurotoxic activities procuced by Duvernoy's gland secretion from the false coral snake (Erythrolamprus bizona Jan, 1863) (Serpentes: Viperidae). Revista Científica FCV-LUZ 13 (5): 371-377.

Lemoine, K.; M.E. Girón; I. Aguilar; L.F. Navarrete \& A. RodríguezAcosta. 2004a. Proteolytic, Hemorrhagic, and Neurotoxic Activities Caused by Leptodeira annulata ashmeadii (Serpentes:Colubridae) Duvernoy's Gland Secretion. Wilderness \& Environmental Medicine 15: 82-89.

Lemoine, K.; L.M Salgueiro \& A. Rodríguez-Acosta. 2004b. Neurotoxic, Hemorrhagic and Proteolytic Activities of Duvernoy's Gland Secretion from Venezuelan Ophistoglyphous Colubrid Snakes in Mice. Veterinary and Human Toxicology 46 (1): 10-14.

Litchield, J.T. \& F. Wilcoxon. 1949. A simplified method of evaluating dose-effect experiments. Jornal of Pharmacology and Experimental Therapeutics 96: 99-113.

Lowry, O.H.; N.J. Rosebrough; A.L. Farr and R.J. Randall. 1951. Protein measurement with the Folin phenol reagent. The Journal of Biological Chemistry 193: 265-275.

MACKeSSY, S.P. 2002. Biochemistry and Pharmacology of Colubrid Snake Venoms. Journal Toxicology-Toxin Reviews 21: 4383.

Mandelbaum, F.R.; M.T. Assakura; A.P. Reicchl \& S.M.T. Serrano. 1998. Philodryas venom metalloproteinases, p.1-2. In: A.J. BARRETT; N.D. RAWLINGS \& J.F. WOESSNER (Eds). Handbook of proteolytic enzymes. New York, Academic Press, XXX+1666p.

Markewell, M.; S.M. Hass; L.L. Bieber \& N.E. Tolbert. 1978. A modification of the Lowry procedures to simplify protein determination in membrane and lipoprotein samples. Analytical biochemistry 87: 206-210.

Navarrete, L.F.; K. Lemoine; A. Rodríguez-Acosta. 1999. Is the Opistoglyph Clelia clelia Duvernoy's Gland Secretion Haemorrhagic in Human? Acta Biologica Venezuelana 19 (3): 19-23.

NicKerson, A.M. \& R.W. HeNDERSON 1976. A case of envenomation by the South American colubrid Philodryas olfersii. Herpetologica 32 (2): 197-198.

Nishioka, S.A. \& P.V.P. Silveira. 1994. Philodryas patagoniensis bite and local envenoming. Revista do Instituto de Medicina Tropical de São Paulo 36 (3): 279-281.

OHSAKA, A. 1979. Hemorrhagic, necrotizing and edema-forming effects of snakesvenoms, p. 481-546. In: C.Y. LeE (Ed.). Snake venoms, handbook of experimental pharmacology. Berlin, Springer, 1129p.

Ovadia, M. 1984. Embryonic development of Duvernoy's gland in the snake Natrix tessellata (Colubridae). Copeia 1984: 516521.

Peichoto, M.E.; L.C. Leiva; L.E. Guaimás moya; L. Rey \& O. Acosta.
2005. Duvernoy's gland secretion of Philodryas patagoniensis from the northeast of Argentina: effects on blood coagulation. Toxicon 45: 527-534.

Peichoto, M.E.; O. Acosta; L. Leiva; P. Teibler; S. Maruñak \& R. Ruíz. 2004. Muscle and skin necrotizing and edema-forming activities of Duvernoy's gland secretion of the xenodontine colubrid snake Philodryas patagoniensis from the north-east of Argentina. Toxicon 44: 589-596.

Peters, J.A. \& Orejas-Miranda, B. 1970. Catalogue of the Neotropical Squamata: Part I. Snakes. Bulletin of United States National Museum 297: 1-347.

Prado-franceschi, J. \& S. Hyslopy. 2002. South American colubrid envenomations. Journal of Toxicology-Toxin Reviews 21: 117-158.

Prado-Franceschi, J.; S. Hyslopy; J.C. Cogo; A.L. Andrade; M.T. Assakura; M.A. Cruz-Höfling \& L. Rodrigues-Simioni. 1996. The effects of Duvernoy's gland secretion from the xenodontine colubrid Philodryas olfersii on striated muscle and the neuromuscular junction: partial characterization of a neuromuscular fraction. Toxicon 34 (4): 459-466.

Prado-Franceschi, J.; S. Hyslopy; J.C. Cogo; A.L. Andrade; M.T. Assakura; A.P. Reichl; M.A. Cruz-Höfling \& L. RodriguesSimioni. 1998. Characterization of a myotoxin from the Duvernoy's gland secretion of the xenodontinae colubrid Philodryas olfersii (green snake): effects on striated muscle and the neuromuscular junction. Toxicon 36 (10): 14071421.

Puorto, G. \& F.O.S. França. 2003. Serpentes não peçonhentas e aspectos clínicos dos acidentes, p. 108-114. In: J.L.C. CARDOso; F.O.S. França; F.H. Wen; C.M.S. Málaque; V. Haddad JR (Eds). Animais peçonhentos no Brasil: biologia, clínica e terapêutica dos acidentes. São Paulo, Sarvier, FAPESP, 468p.

Ribeiro, L. A.; G. Puorto \& M.T. Jorge. 1994. Acidentes por serpentes do gênero Philodryas: avaliação de 132 casos. Revista da Sociedade Brasileira de Medicina Tropical 27 (Supl. 1): 87.

Ribeiro, L.A.; G. Puorto \& M.T. Jorge. 1999. Bites by colubrid snake Philodryas olfersii: a clinical and epidemiological study of 43 cases. Toxicon 37 (1999): 943-948.

Rocha, M.M.T.; S.R. Travaglia-Cardoso \& C. Sato. 2003. Reports of Human Snake Accidents Caused by Philodryas olfersii. Memórias do Instituto Butantan 60: 142.

Rocha, M.M.T.; D. Paixão-Cavalcante; D.V. Tambourgi \& M.F.D. FurTado. 2006. Duvernoy's gland secretion of Philodryas olfersii and Philodryas patagoniensis (Colubridae): Neutralization of local and systemic effects by commercial bothropic antivenom (Bothrops genus). Toxicon 47: 95-103.

Rosenberg, H.I.; A. Bdolah \& E. Kochva. 1985. Lethal factors and enzymes in the secretion from Duvernoy's gland of three colubrid snakes. The Journal of Experimental Zoology 233: 5-14.

Rosenfeld, G. 1971. Symptomatology, pathology, and treatment of snake bites in South America, p. 345-384. In: W. BuCHERL; 
E.E. Buckley \& V. Deulofeu (Eds). Venomous animals and their venoms. New York, Academic Press, vol. 2, 687p.

Salomão, M.G.; A.B.P. Albolea \& S.M. Almeida-Santos. 2003. Colubrid snakebite: a public health problem in Brazil. Herpetological Review 34 (4): 307-312.

Salomão, M.G. \& M. Di Bernardo. 1995. Philodryas olfersii: uma cobra comum que mata. Caso registrado na área da $8^{\text {a Dele- }}$ gacia Regional de Saúde. Arquivos da Sociedade de Zoológicos do Brasil (14-16): 21.

Sanchez, E.F.; T.V. Freitas; D.L. Ferreira-Alves; D.T. Velarde; M.R. Diniz; M.N. Cordeiro; G. Agostini-Cotta \& C.R. Diniz. 1992. Biological activities of venoms from south american snakes. Toxicon, Oxford, 30(1): 95-103.

Santos-Costa, M.C.; A.B. Outeiral; F. D’Agostini \& L. Cappeliari. 2001. Freqüência de acidentes ofídicos na região da grande Porto Alegre e cidades próximas, RGS, Brasil. Comunicação do Museu de Ciências e Tecnologia da PUCRS, Série Zoologia, 14 (1): 89-93.

Silva, M. V. \& M. A. Buononato. 1983. Relato clínico de envenenamento humano por Philodryas olfersii. Memórias do Instituto Butantan 47/48: 121-126. [1984]

Silveira, P.V.P. \& S.A. Nishioka. 1992. Non-venomous snake bite and snake bite without envenoming in a brazilian teaching Hospital. Analysis of 91 cases. Revista do Instituto de Medicina Tropical de São Paulo 34: 499-503.

Tanjoni, I.; D. Butera; P.J. Spencer; A.H. Takehara; I. Fernandes \& A.M. Moura-DA-SiLVA. 2003. Phylogenetic conservation of a snake venom metalloproteinase epitope recognized by monoclonal antibody that neutralizes hemorrhagic activity. Toxicon 42: 809-816.

Theakston, R.D.G. \& H.A. Reid. 1983. Development of simple standard assay procedures for the characterization of snake venoms. Bulletin of the World Health Organization 61: 949-956.

VANZOLINI, P. 1986. Levantamento herpetológico da área do estado de Rondônia sob influência da rodovia BR 364 . Brasília, CNPq, Relatório de pesquisa, 50p.

VEST, D.K. 1988. Some effects and properties of Duvernoy's gland secretion from Hypsiglena torquata texana (Texas night snake). Toxicon 26 (4): 417-419.

VeST, D.K.; S.P. MaCKessY \& K.V. KaRdong. 1991. The unique Duvernoy's secretion of the brown tree snake (Boiga irregularis). Toxicon 29 (4/5): 532-535.

Villarroel, M.S.; F. Zelante; R. Rolim Rosa \& R.S. Furlanetto. 1978. Padronização da titulação da atividade tóxica de venenos botrópicos, em camundongos. Memórias do Instituto Butantan 42/43: 311-323. [1979]

WARRELL, D.A. 2004. Snakebites in Central and South America: Epidemiology, Clinical Features, and Clinical Management, p. 709-761. In: J.A. Campbell \& W.W. Lamar (Eds). The Venomous reptiles of the western hemisfephere. London, Comstok, vol. 2, $6^{\text {th }}$ ed., $425 \mathrm{p}$.

Weinstein, S.A.; D. Chiszar; R.C. Bell \& L.A. Samith. 1991. Lethal potency and fractionation of Duvernoy's secretion from the brown tree snake, Boiga irregularis. Toxicon 29 (4/5): 401-407.

Weinstein, S.A. \& K.V. Kardong. 1994. Properties of Duvernoy's secretions from opisthogliphous and aglyphous colubrid snakes. Toxicon 32 (10): 1161-1185.

YAMAKAWA, S.A.; M. NOZAKI \& Z. HOKAWA. 1976. Fractionation of sakishimahabu (Trimeresurus elegans) venom and lethal, hemorrhagic and edema-formin activities of the fractins, $\mathrm{p}$. 97-109. In: A. OHSAKA; K. HAYASHI; Q.Y. SAWAY (Eds). Animal, plant and microbial toxins. New York, Plenum, vol. 1, $\mathrm{XXV}+555 \mathrm{p}$.

Recebido em 28.IX.2006; aceito em 10.V.2007.

Revista Brasileira de Zoologia 24 (2): 410-418, junho 2007 